Prepared in cooperation with the North Pacific LCC

\title{
Ecological Context for the North Pacific Landscape Conservation
} Cooperative

Andrea Woodward Audrey Taylor Anne Weekes

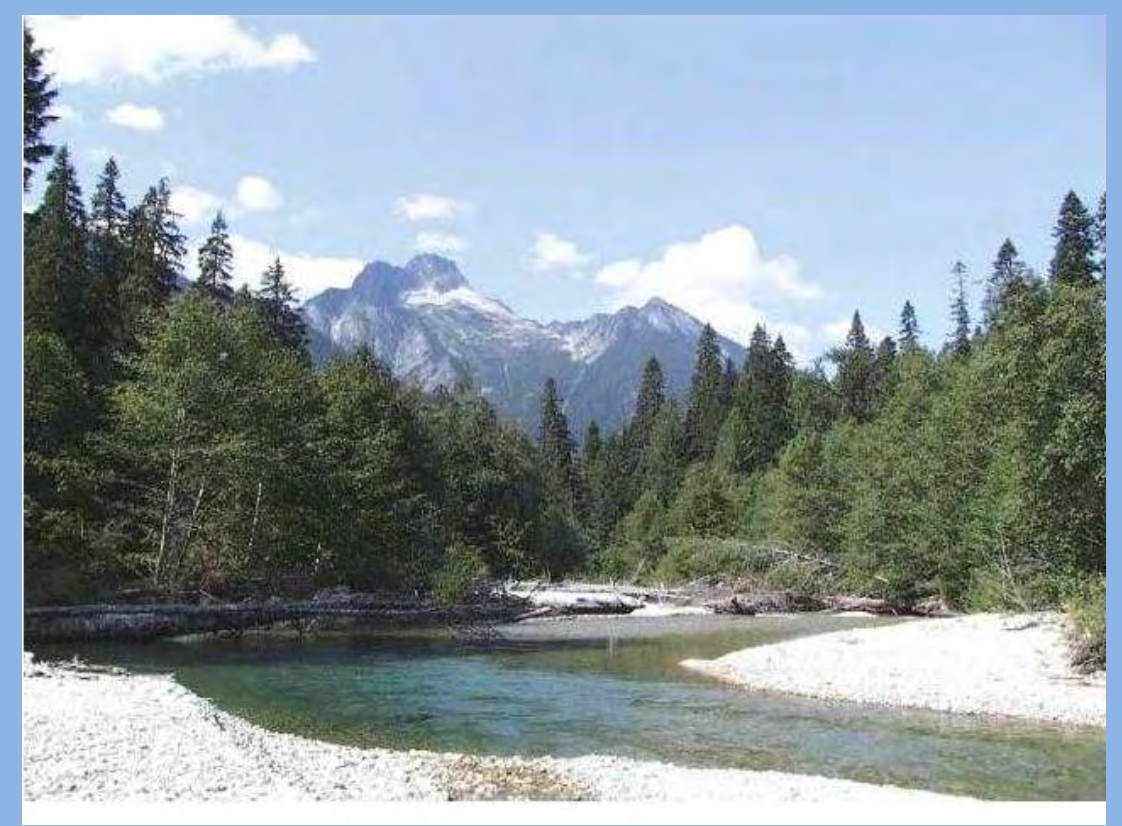

U.S. Geological Survey Open-File Report 2012-1211 


\section{BACKGROUND}

The North Pacific Landscape Conservation Cooperative (NPLCC) encompasses the temperate coastal rainforest and extends from the coastal mountains to the near-shore from the Kenai Peninsula, Alaska to Bodega Bay, California. The area spans multiple agency, state, and international boundaries over more than 22 degrees of latitude, including a wide range of type and intensity of human land-use activities. Development of NPLCC goals and administrative structures will be facilitated by a shared ecological context for discussing this expansive, diverse, and complex landscape.

In support of activities to organize the NPLCC, we provided conceptual models to describe the ecological structure of the NPLCC. Recognizing that the boundaries of LCCs were primarily based on Level 2 of the hierarchical ecoregional classification of Omernik (Comission for Environmental Cooperation 1997), we used nested Level 3 ecoregions to define subregions within the NPLCC. Rather than develop conceptual models for all nine constituent subregions, we opted to consider five groups: Puget-Georgia Basin Lowland and Willamette Valley, Alaska-British Columbia Coast, Alaska-British Columbia Mountains, Klamath-Olympic-Cascade Mountains, and Washington-Oregon-Northern California Coast. At the conclusion of the project, we felt that the close relationship between mountain and coastal areas support combining them to create three major subregions: Alaska-British Columbia coast and mountains, Washington-Oregon-Northern California coast and mountains, and the lowlands of the Georgia Basin and Willamette Valley.

The following figures present the Omernik Level 3 ecoregions comprising the NPLCC; how the ecoregions were grouped to create conceptual models; and conceptual models for each group. The five models each consist of a table listing resources, stressors, potential climate change impacts; a landcover map; and a cartoon to summarize the table and evoke the landscape. A final figure summarizes resources, stressors, and climate change impacts that are common across the NPLCC.

\section{Cited:}

Commission for Environmental Cooperation,1997, Ecological regions of North America: toward a common perspective: Montreal Canada, Commission for Environmental Cooperation. 


\section{Ecoregions of the NPLCC}

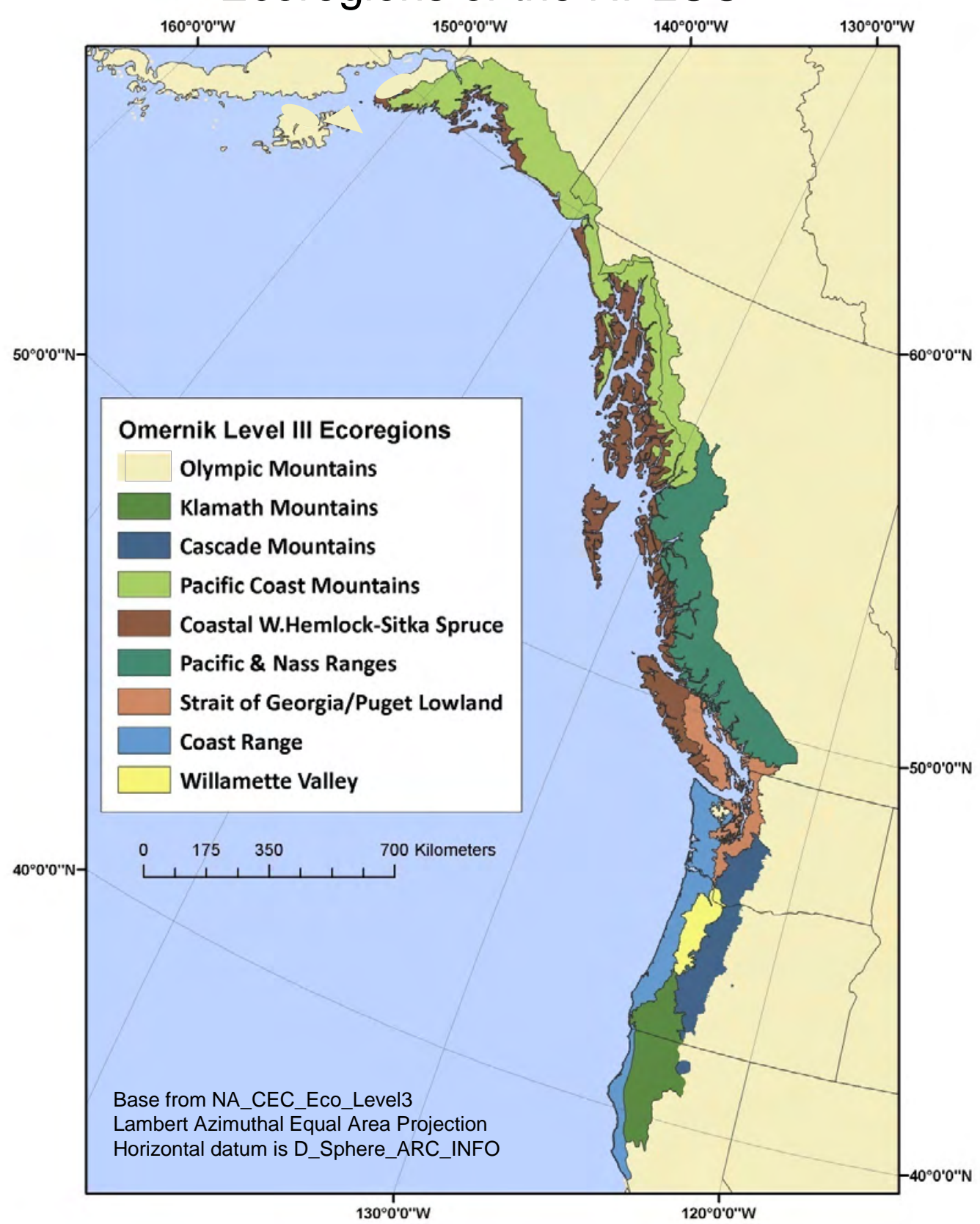


Ecoregional groups for which conceptual models were developed

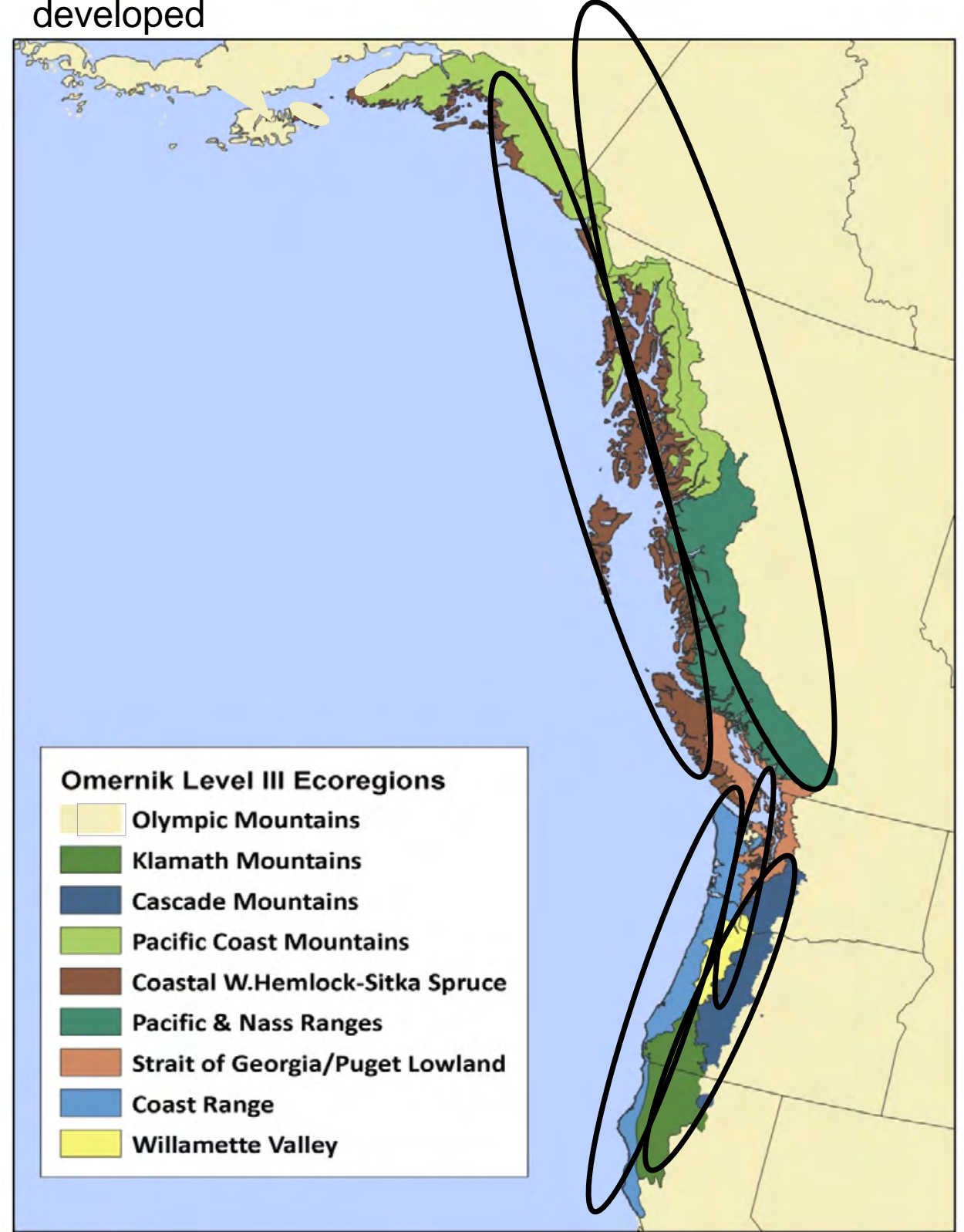


PUGET-GEORGIA BASIN LOWLANDS \& WILLAMETTE VALLEY

\begin{tabular}{|c|c|c|}
\hline $\begin{array}{l}\text { Valued Human, Ecological, and Cultural } \\
\text { Resources }\end{array}$ & Existing Stressors & $\begin{array}{l}\text { Current and Potential Climate Change } \\
\qquad \text { Impacts }\end{array}$ \\
\hline 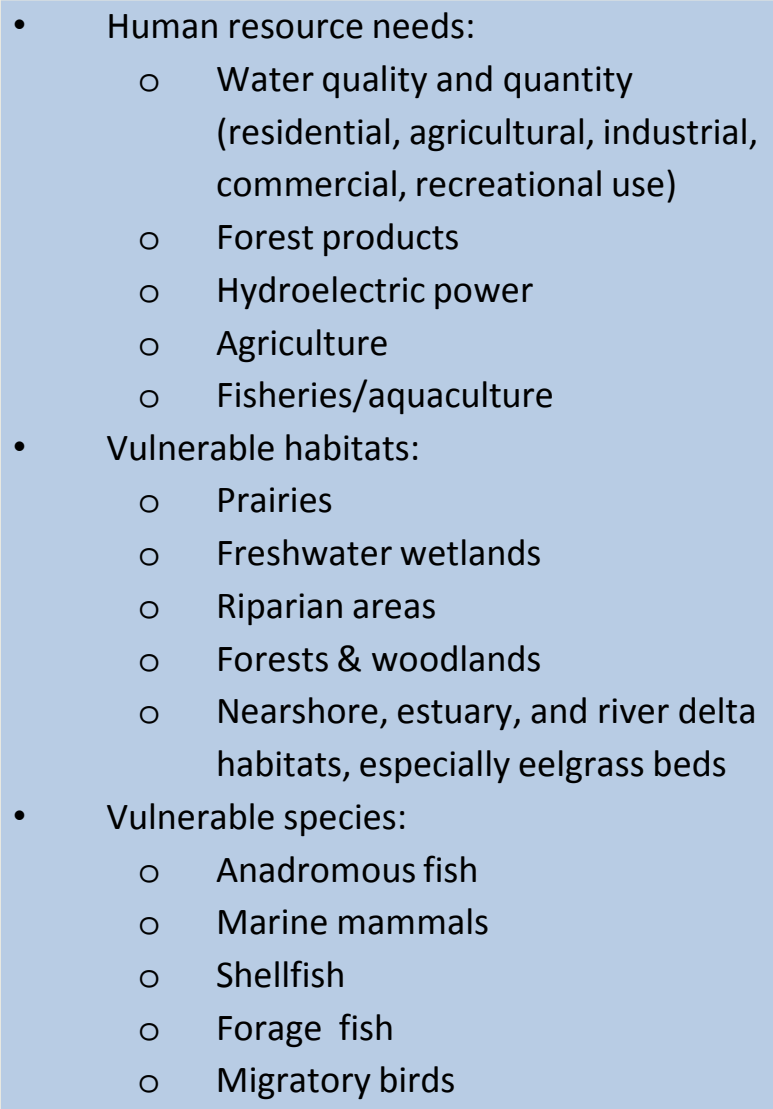 & $\begin{array}{l}\text { Changes in land cover/land use: } \\
\begin{array}{c}\text { Increased water withdrawal } \\
\text { (urban, agriculture, mining) } \\
\text { Larger floods/ } \\
\text { aggradation/channel capacity } \\
\text { Water quality/ } \\
\text { turbidity/increased fine } \\
\text { sediment loads } \\
\text { Habitat alteration, loss, } \\
\text { fragmentation } \\
\text { Urbanization: } \\
\text { O Increased impermeable } \\
\text { surface/ heat islands } \\
\text { o Storm water, waste water } \\
\text { pollutants } \\
\text { Emissions - particulates, } \\
\text { persistent organic pollutants } \\
\text { Shoreline armoring \& development }\end{array}\end{array}$ & $\begin{array}{l}\text { - Hydrologic regime changes: } \\
0 \quad \text { Sediment dynamics changes in } \\
\text { rivers, estuaries and shorelines } \\
\text { - } \quad \text { Increased atmospheric temperatures: } \\
0 \quad \text { Extreme/unpredictable weather } \\
0 \quad \text { Plant \& animal stress } \\
0 \quad \text { Forest disturbance increase } \\
\text { Sea level rise (exacerbated by } \\
\text { subsidence in Puget Sound): } \\
\text { o Coastal flooding \& salt water } \\
\text { intrusion } \\
\text { Ocean acidification }\end{array}$ \\
\hline
\end{tabular}

\section{Selected References}

Puget Sound Partnership, 2010, State of the Sound Report: Olympia, Washington, Publication no. PSP09-08.

Alberti, M. and Waddell, P., 2006, An integrated urban development and ecological simulation model: Integrated Assessment v. 1, p. $215-227$.

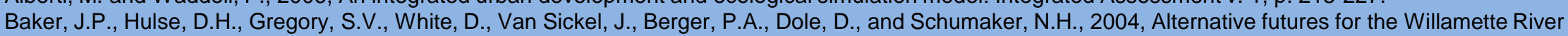
basin, Oregon: Ecological Applications, v. 14, p. 313-324.

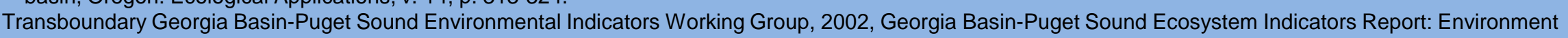
Canada web site at http://www.ec.gc.ca/Publications. (Accessed September 19, 2012.)

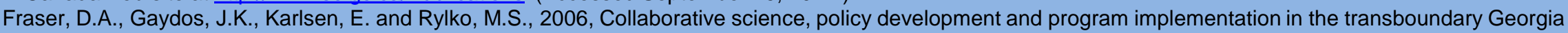
Basin/Puget Sound ecosystem: Environmental Monitoring and Assessment v. 113, p. 49-69.

University of Washington Climate Impacts Group web site at http://cses.washington.edu/cig. (Accessed September 19, 2012) 


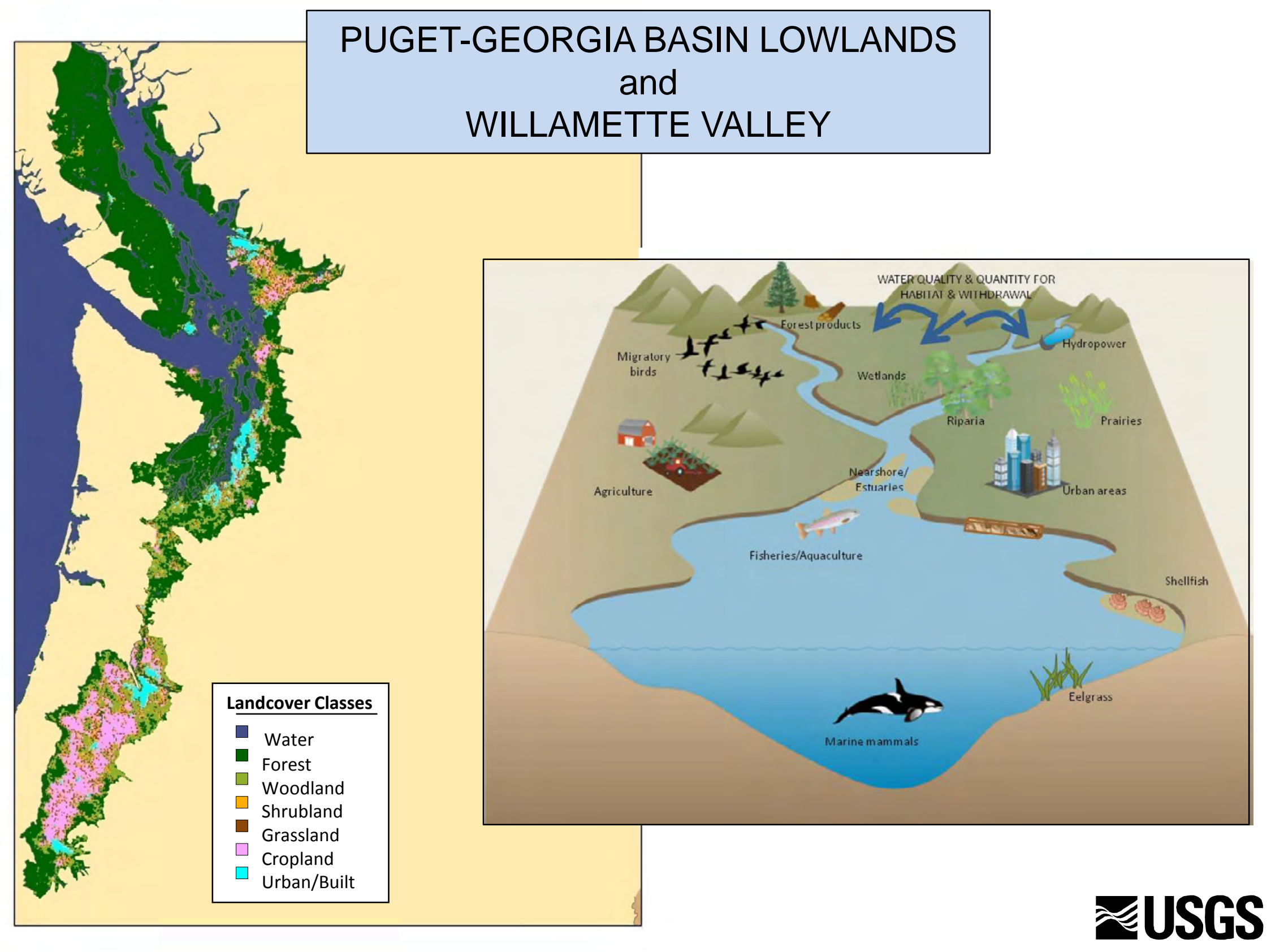




\section{ALASKA-BRITISH COLUMBIA COAST}

\begin{tabular}{|c|c|c|}
\hline $\begin{array}{l}\text { Valued Human, Ecological, and Cultural } \\
\text { Resources }\end{array}$ & Existing Stressors & Current and Potential Climate Change Impacts \\
\hline 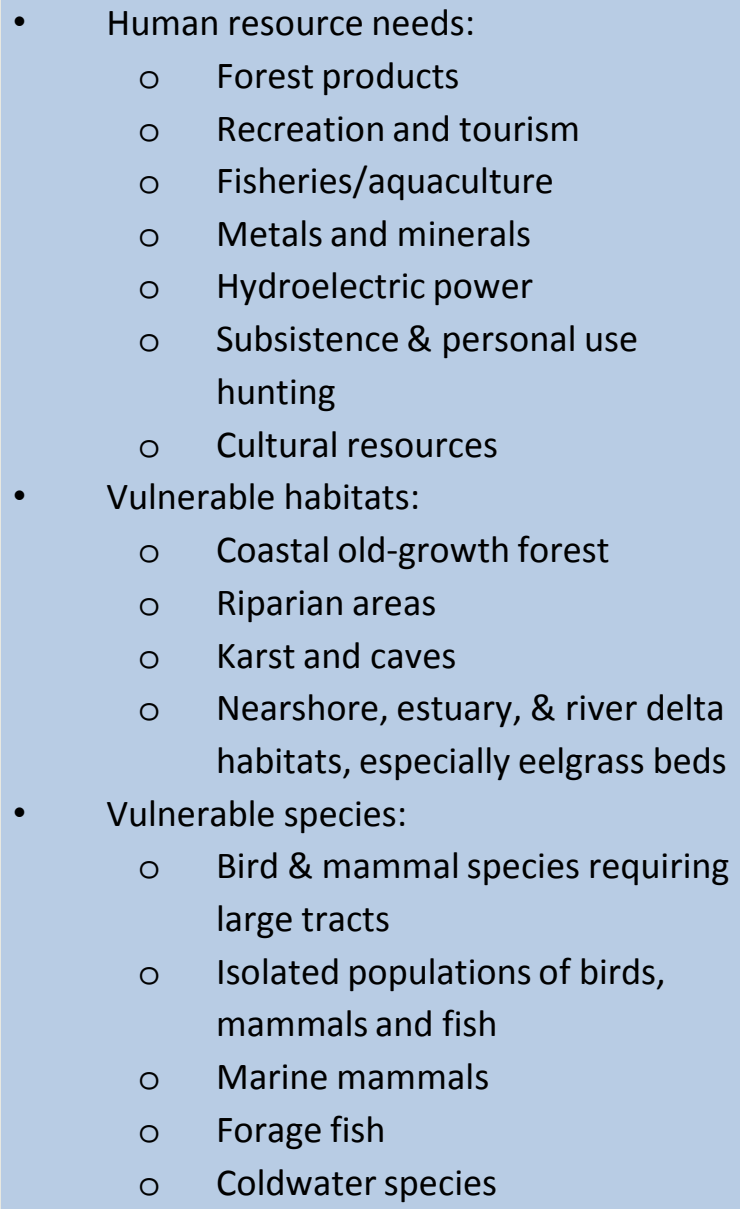 & $\begin{array}{l}\text { - Timber harvest: } \\
0 \quad \text { Habitat fragmentation or loss } \\
0 \quad \text { Salmon habitat degradation } \\
0 \quad \text { Subsistence species loss } \\
\text { - } \quad \text { evelopment (including for } \\
\text { transportation): } \\
\text { o Habitat fragmentation } \\
\text { o Fish passage impediments } \\
\text { o Fish \& wildlife consumption } \\
\text { increase } \\
\text { - Wetland and nearshore habitat } \\
\text { - } \quad \text { alteration or loss } \\
\text { Hydroelectric projects } \\
\text { o Hydrology alteration } \\
\text { - Fish passage impediments } \\
\text { Alternative energy (currents \& tidal) } \\
\text { Introduced pathogens: } \\
\text { o Fish \& wildlife population and } \\
\text { Air \& water pollution (from economic } \\
\text { activity) }\end{array}$ & $\begin{array}{l}\text { - Hydrologic regime changes: } \\
0 \quad \text { Longer ice/snow-free season } \\
0 \quad \text { Snowpack depth changes } \\
\text { - Increased atmospheric temperatures: } \\
0 \quad \text { Forest pathogen distribution } \\
\text { increased } \\
\text { - Sea level rise (mitigated by isostatic } \\
\text { rebound): } \\
0 \quad \text { Shoreline erosion } \\
0 \quad \text { Estuary and aquifer salinity } \\
\text { - increases } \\
\text { Ocean acidification } \\
\text { Depth of ocean stratification }\end{array}$ \\
\hline
\end{tabular}

Selected references

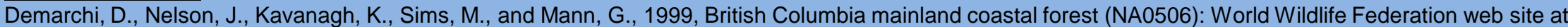
http://ecoregions/na0506. (Accessed September 20, 2012.)

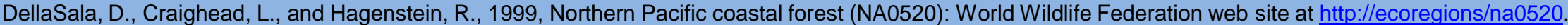
(Accessed September 20, 2012.)

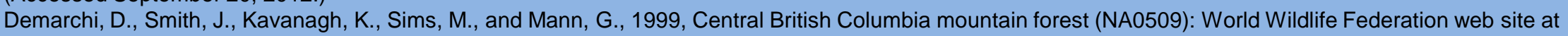
http://ecoregions/na0509. (Accessed September 20, 2012.)

University of Washington Climate Impacts Group web site at http://cses.washington.edu/cig. (Accessed September 19, 2012) 


\section{ALASKA-BRITISH COLUMBIA COAST}
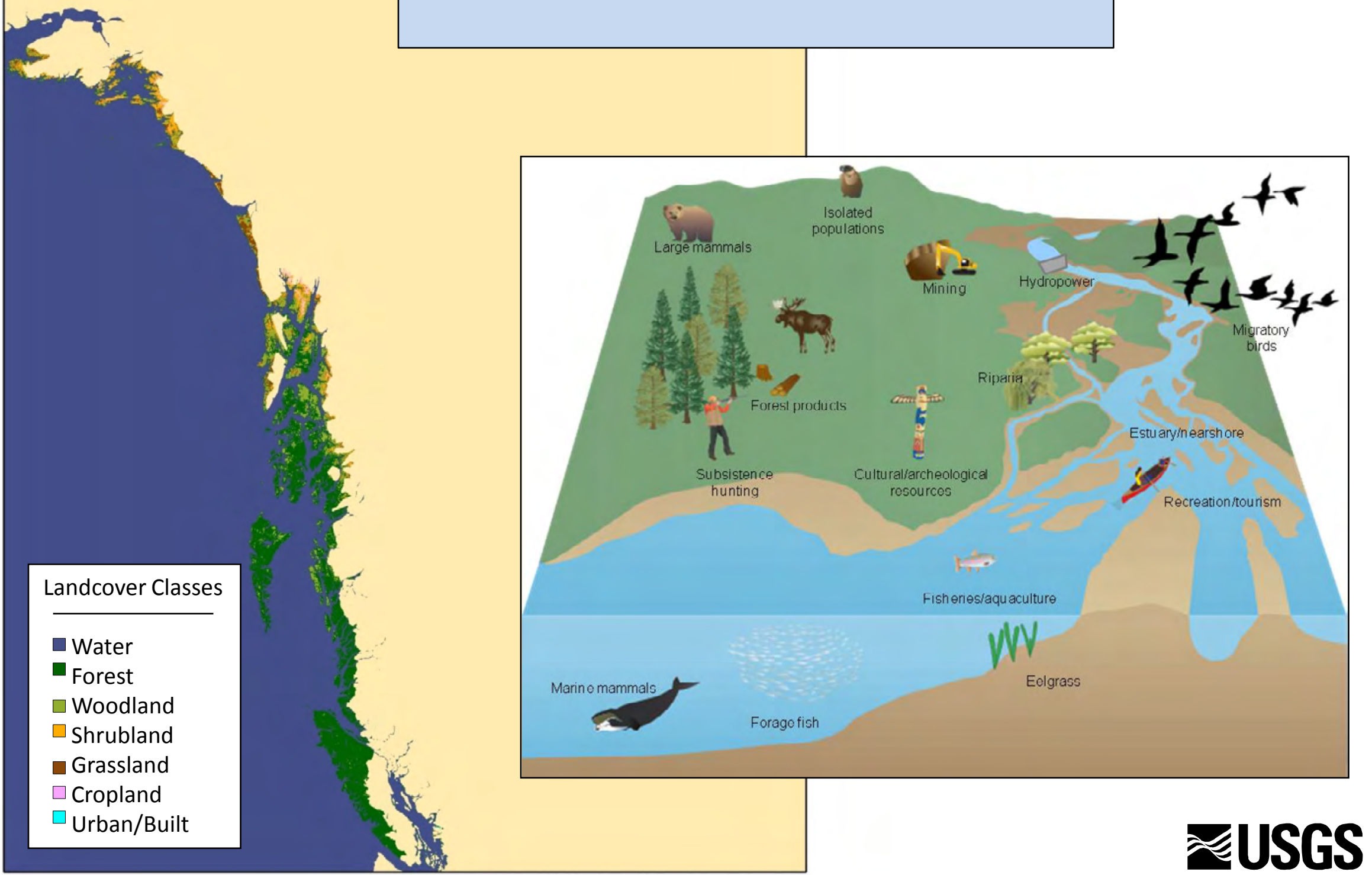
ALASKA-BRITISH COLUMBIA MOUNTAINS

Valued Human, Ecological, and Cultural Resources

- Human resource needs:

o Metals and minerals

o Electric power (hydro-, gas/oil, wind)

o Forest products

o Subsistence/personal use hunting \& gathering

o Recreation \& tourism

- Vulnerable habitats:

o Coastal old-growth forest

o Subalpine forest

o Alpine tundra

o Eastern slope/Nass Basin forest

o Riparian areas

o Mountain lakes

- Vulnerable species:

o Bird \& mammal species requiring large tracts

o Isolated populations of birds, mammals and fish

o Coldwater and mountain species

o Anadromous fish
Current and Potential Climate Change Impacts

Timber harvest and mining:

o Habitat fragmentation or loss

o Salmon habitat degradation

- Development (including for transportation):

o Habitat fragmentation

o Fish passage impediments

o Fish \& wildlife consumption increase

o Wetland alteration or loss

o Contaminants

- Hydroelectric projects

o Hydrology alteration

o Fish passage impediments

- Introduced pathogens:

o Fish \& wildlife population and habitat loss
- Hydrologic regime changes:

o Glacier melt

o Ice/snow-free season changes

o Snowpack depth changes

o Soil water storage changes (drying of muskeg)

Increased atmospheric temperatures:

o Forest pathogen distribution increase

- Changes in plant community distribution

o Alpine replaced by forest

o Yellow cedar decline

o Wetland community decline

Selected references

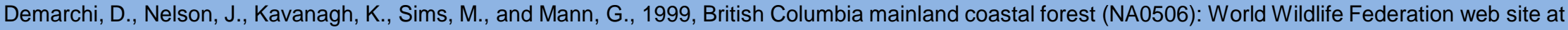
http://ecoregions/na0506. (Accessed September 20, 2012.)

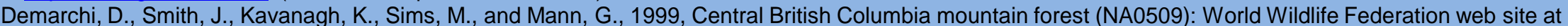
http://ecoregions/na0509. (Accessed September 20, 2012.)

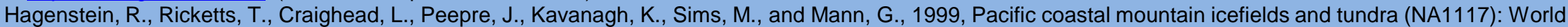
Wildlife Federation web site at http://ecoregions/na1117. (Accessed September 20, 2012.)

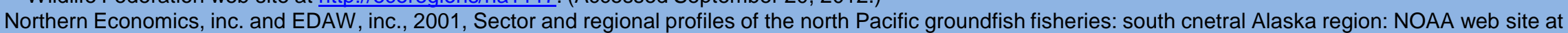
http://www.fakr.noaa.gov/npfmc/PDFdocuments/resources/SectorProfiles/Southcentral\%20Alaska\%20Profile.pdf. (Accessed September 18, 2012.) 


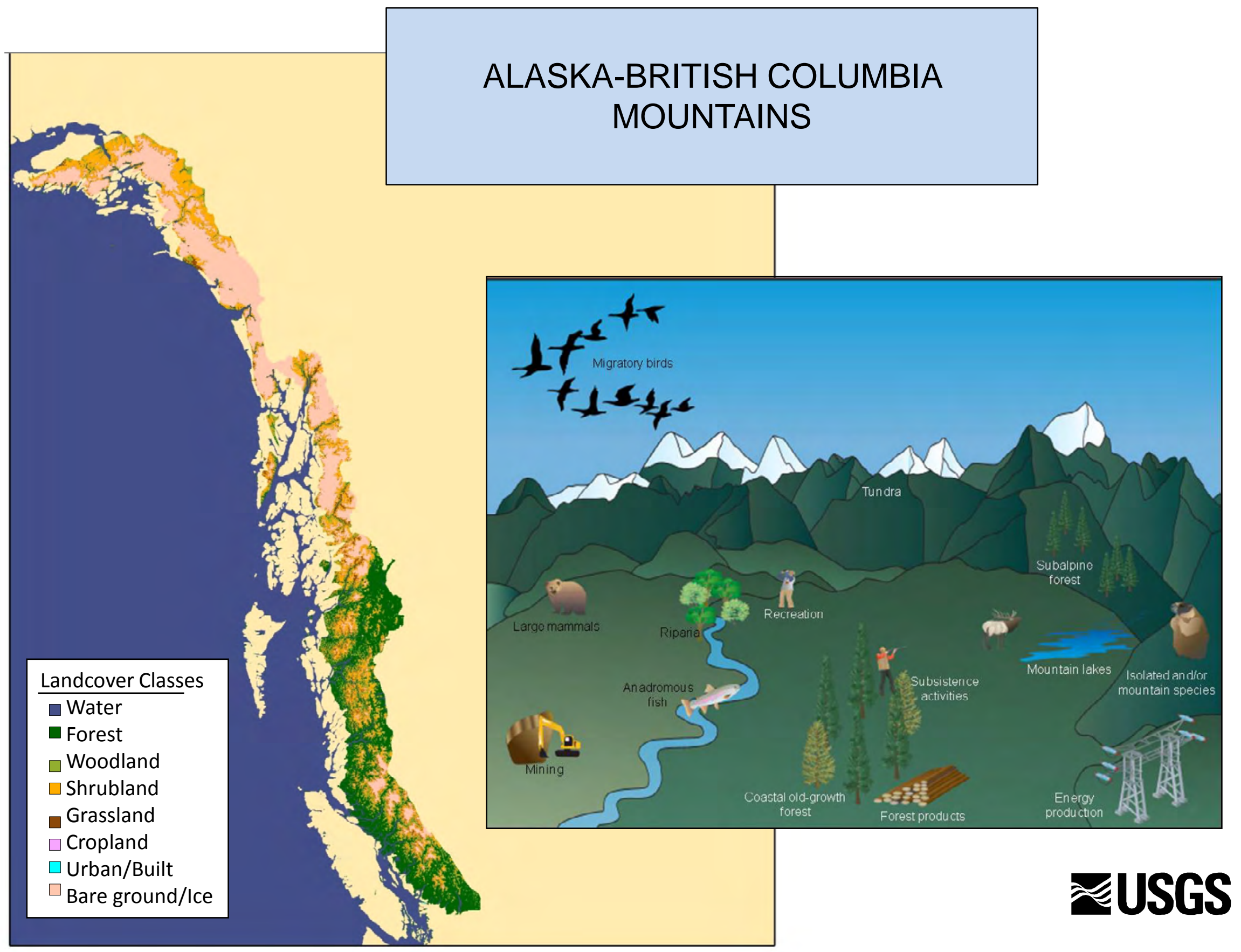


KLAMATH-OLYMPIC-CASCADE MOUNTAINS

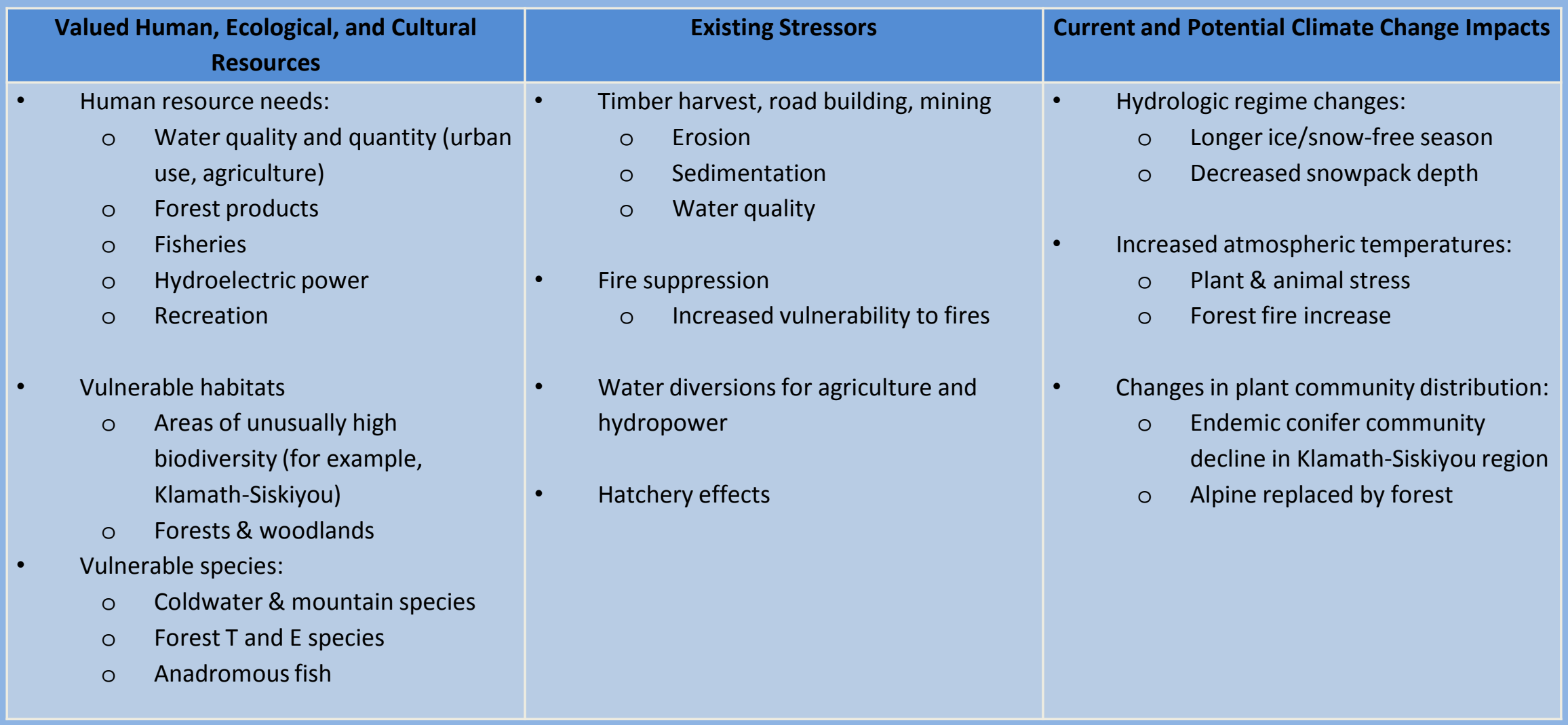

Selected references

Olson, D., Noss, R., Orians, G., Stritholt, J., Williams, C., and Sawyer, J., 1999, Klamath-Siskiyou forest (NA0516): World Wildlife Federation web site at http://ecoregions/na0516. (Accessed September 20, 2012.)

DellaSala, D., Stritthold, J., Noss, R., and Orians, G., 1999, Central and southern Cascades (NA0508): World Wildlife Federation web site at http://ecoregions/na0508. (Accessed September 20, 2012.)

Damschen, E.I., Harrison, S., and Grace, J.B., 2010, Climate change effects on an endemic-rich flora: resurveying Robert H. Whittaker's Siskiyou sites (Oregon, USA): Ecology, v. 91, p. 3609-3619.

Mid-Klamath Watershed Council web site at http://www.mkwc.org/programs/firefuels/Klamath\%20Fire\%20Ecology\%20Symposium/index KFES.htm. (Accessed September 18, 2012.) 


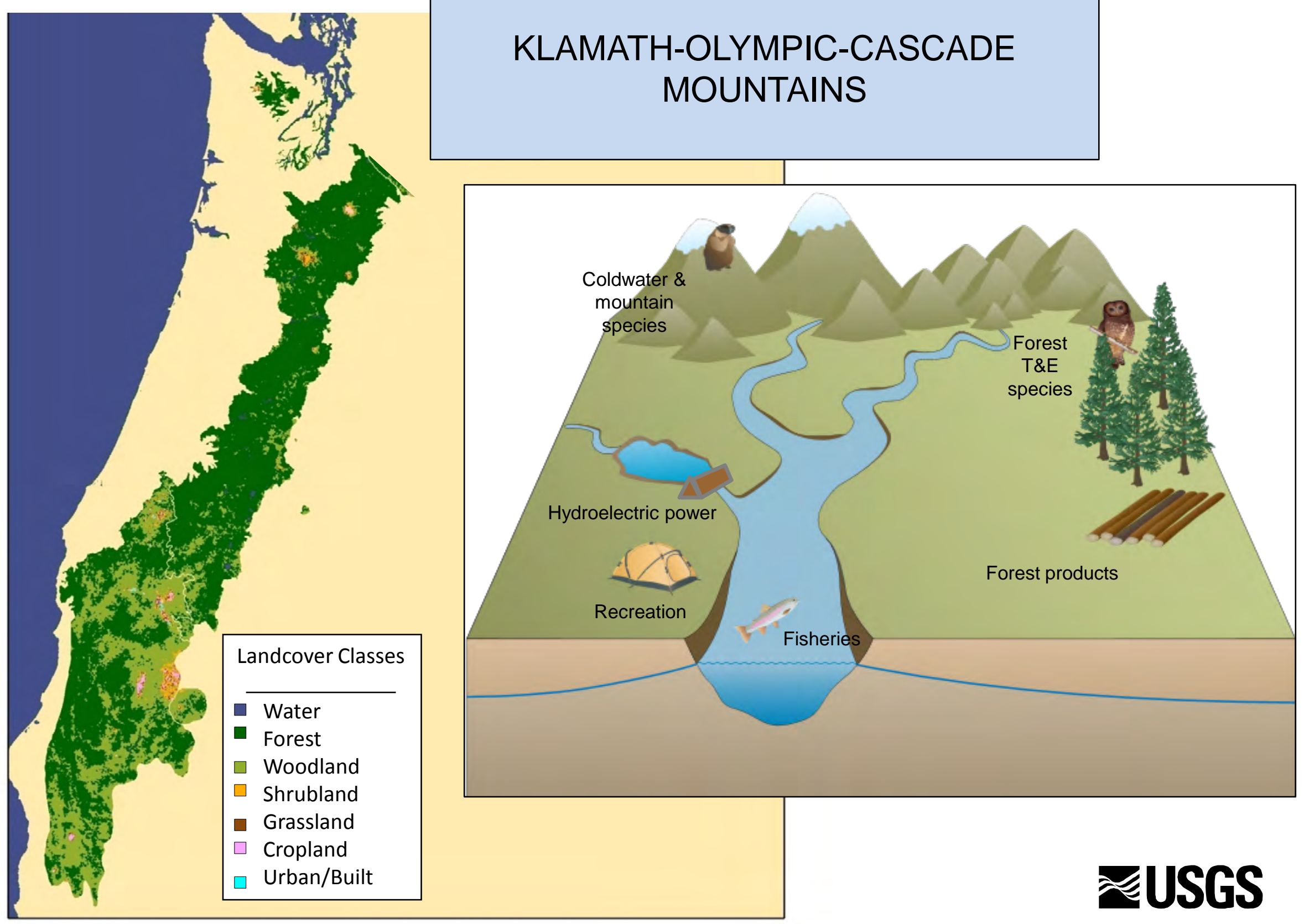


WASHINGTON-OREGON-NORTHERN CALIFORNIA COAST

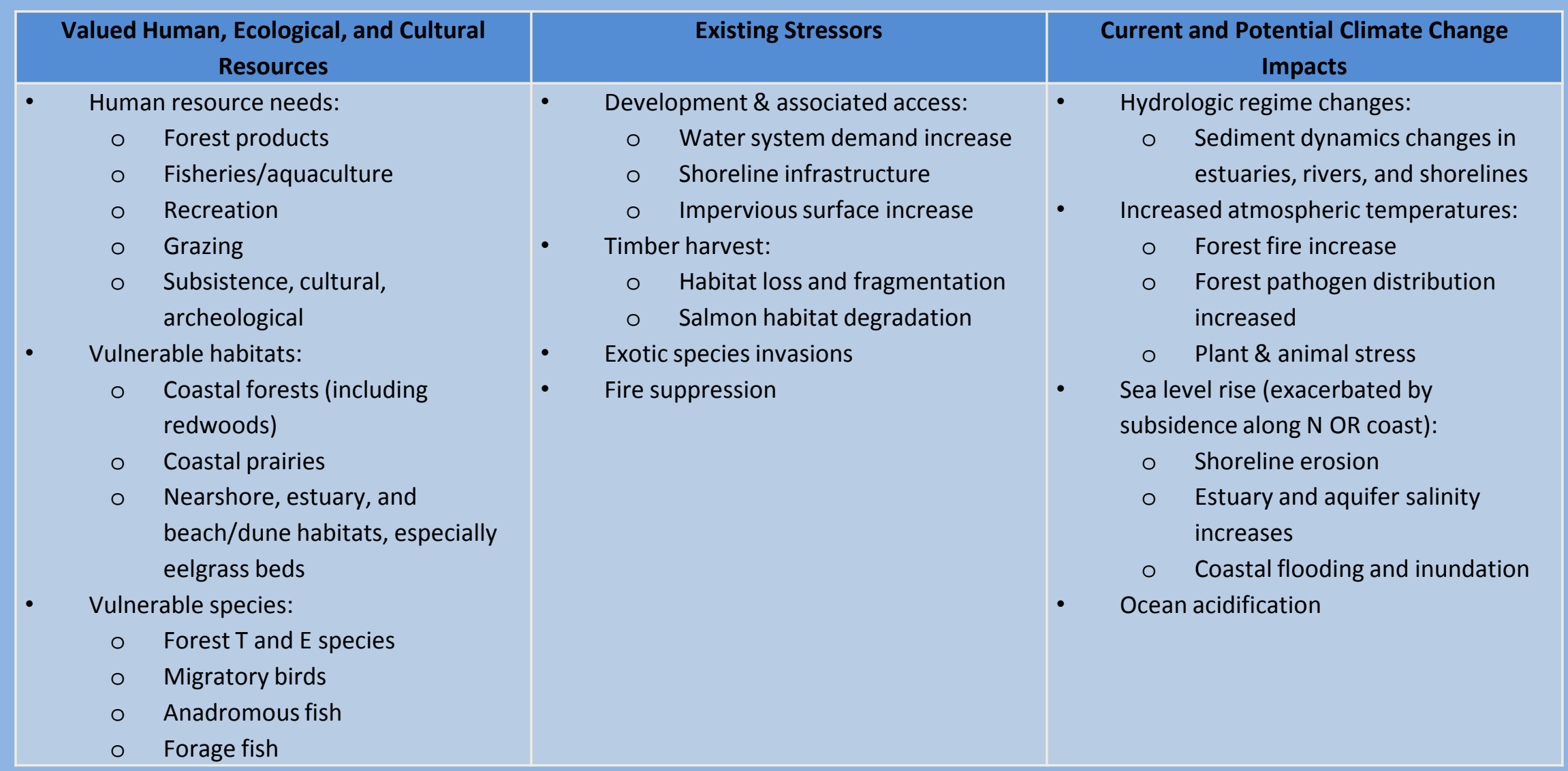

Selected references

Noss, R., Strittholt, G., Orians, G., Adams, J., Kavangh, K., Sims, M., and Mann, G., 1999, Central Pacific coastal forest (NA0510): World Wildlife Federation web site at http://ecoregions/na0510. (Accessed September 20, 2012.)

Olson, D. and Sawyer, J., 1999, Northern California coastal forests (NA0519): World Wildlife Federation web site at http://ecoregions/na0519. (Accessed September 20, 2012.) 


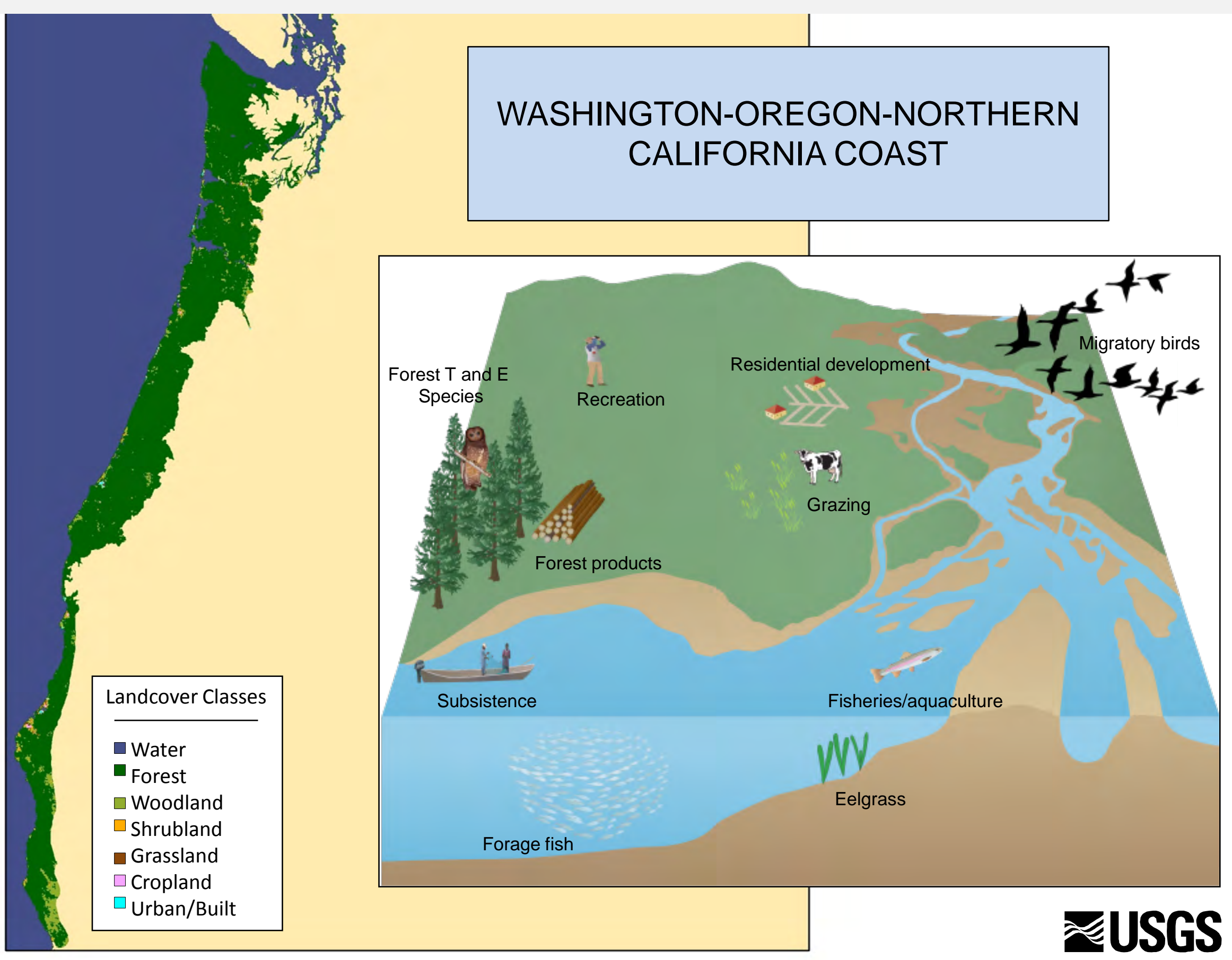




COMMON THEMES ACROSS NPLCC
Valued human, ecological, and cultural
resources
- Forest products
- Old growth forests
- Freshwater quality, timing, availability
- Anadromous fish populations
- Migratory birds
- Carbon sequestration capacity
- Cultural resources
- Habitat connectivity
- Near shore / coastal / estuarine habitats
- Community stability / human well-being
- Recreation / tourism
Climate-related stressors and potential
climate change impacts
- Climate change
- Infrastructure development
- Energy development
- Invasive species
- Sea level rise
- Ocean acidification
- Ocean current changes
- Food web dynamics
- Phenological mismatches
- Disturbed regimes
- High sensitivity to land-sea interactions
- Global economic dynamics

